\title{
Outage Performance for DF Two-Way Relaying with Co-Channel Interference over Nakagami- $m$ Fading
}

\author{
Jinhong Fan ${ }^{1,2}$ and Chaowei Yuan ${ }^{1}$ \\ ${ }^{1}$ School of Information and Communication Engineering, Beijing University of Posts and Telecommunications, \\ Beijing, 100876 - P.R.China \\ [e-mail: qfjh0922@163.com] \\ ${ }^{2}$ College of Electronic Information Engineering, North China University of Technology, \\ Beijing, 100144 - P.R.China \\ *Corresponding author: Jinhong Fan
}

Received June 4, 2015; revised August 19, 2015; accepted September 4, 2015; published November 30, 2015

\begin{abstract}
In this paper, we investigate the outage performance of a two-way decode-and-forward relaying network in an interference-limited Nakagami- $m$ fading environment. More specifically, assuming the presence of Nakagami- $m$ faded multiple co-channel interferers at the source/destination terminals, the closed-form approximate expression for the outage probability is derived by using moment-based estimators attaining the appropriate Nakagami- $m$ fading parameter. Simulation results demonstrate that our analytical result is in excellent agreement with the Monte Carlo simulation.
\end{abstract}

Keywords: Two-way relaying, outage probability, decode-and-forward, co-channel interference, Nakagami- $m$ fading

This research was supported by National Natural Science Foundation of China under grant 60872149 and Scientific Research Common Program of Beijing Municipal Commission of Education under grant No.201510009008. 


\section{Introduction}

Relaying technologies can improve throughout and increase communication range. They have emerged as an effective means for exploiting spatial diversity [1,2]. Channel capacity is increased [3] and full diversity gain can be achieved [4] by sharing antennas amongst users via relaying. The capacity of relay channels was first studied in [5] where Cover et al. considered the classical three-node relay network and derived the channel capacity. Two protocols for one-way relay networks, known as decode-and-forward (DF) and amplify-and-forward (AF) relaying, were proposed in [6]. Due to the capacity loss of the pre-log factor of 1/2 [7] in half-duplex relaying, two-way relaying has been introduced, e.g., [8-10]. Until now, the performance analysis and different transmission schemes for AF and DF two-way relaying network have been well investigated in the literature. When a network coding approach [11] is applied to relay networks, two-way DF [8] and $\mathrm{AF}$ [9] relaying schemes were proposed. It has been illustrated in [10] that both DF and AF two-way relaying protocols can fully recover the spectral efficiency loss. For this reason, two-way relaying networks have attracted much attention in wireless communications. The rate performance of two-way relaying network has been extensively studied in recent years. The achievable rate regions for half-duplex two-way relaying network were studied in [12,13]. In [14], the exact outage probabilities for a three-node two-way relaying network using $\mathrm{AF}$ and $\mathrm{DF}$ schemes were presented and an adaptive AF/DF scheme was proposed to achieve the optimal outage performance. The two-way relaying technology was combined with small cell $[15,16,17]$ technology and Multiple-Input Multiple-Output (MIMO) [18] technology.

The co-channel interference is inevitable in wireless communications. The performance analysis of one-way relaying network has turned the focus to interference-limited channels [19-21]. One-way relaying system with noisy relay and interference-limited destination was examined and the closed-form outage probabilities were derived for both DF and AF protocols [19]. One-way relaying system with multiple interferers over Rayleigh fading channels was studied in [20]. The exact outage probability of the DF one-way relaying system with unequal-power interferers using opportunistic relay selection was provided in [21]. A DF one-way relaying network using opportunistic relaying selection was investigated in [22], where the outage-optimal relay selection was proposed and the closed-form outage probabilities were derived in a multi-cell environment. A DF two-way relaying network with co-channel interferences was investigated in [23], where a tight approximate expression of the average symbol error rate was derived in closed-form. In [24], the exact symbol error probability of two-way DF relaying network in the presence of a finite number of co-channel interferes and noise at the relay and the source codes in Rayleigh fading environment was studied. The interference limits were considered in cognitive femtocells system [25].

On the other hand, the performance of the relaying network based on the Nakagami- $m$ fading channels has been investigated recently. $\operatorname{In}[26-28]$, the performance of two-way interference-limited AF relaying systems with over Nakagami-m fading channels was investiaged. In [29], the outage performance of dual-hop DF cooperative systems in the interference-limited Nakagami- $m$ fading environment was investigated. The symbol error probability of a two-way relay-based communication system with opportunistic relay selection in Nakagami- $m$ fading environments was studied in [30]. In this letter, we consider an interference-limited Nakagami-m fading environment in the investigation of the outage performance of a DF two-way relaying network with multiple interferers. 
The rest of this paper is organized as follows. The system model is described in Section 2. In Section 3, we investigate the outage probability of the DF two-way relaying system with multiple interferers in Nakagami- $m$ fading environment. In Section 4, some numerical results are given to verify our analysis. Finally, Section 5 concludes the paper.

Notations-we denote $P\{B\}$ as the probability of a random event $B$, while $E\{B\}$ returns the expected value of the input random variable or event. $F_{B}(x)$ and $f_{B}(x)$ are, respectively, the cumulative density function and the probability density function of $B .\left(\begin{array}{l}x \\ y\end{array}\right)$ represents the binomial coefficient, whereas $\Gamma(n, x)=\int_{x}^{+\infty} t^{n-1} e^{-t} d t$ and $\Gamma(n)=\int_{0}^{+\infty} t^{n-1} e^{-t} d t$ are the upper Gamma and the Euler Gamma functions, respectively.

\section{System Model}

The two-way relaying system model with co-channel interference is shown in Fig. 1, where two mobile terminals, $S_{1}$ and $S_{2}$, at the edges of two different cells exchange message with the aid of a relay node $R$, assuming that the direct path between them is broken. The source nodes, $S_{1}$ and $S_{2}$, are at the cell-edges and suffer from interference from other mobile/relay nodes in adjacent cells, while the relay node $R$ is free from interference. We assume that interference is the dominating factor limiting the performance. The noises at $S_{1}$ and $S_{2}$ are ignored, whilst $R$ is noise-limited. The forward and backward channels are reciprocal, with $\alpha_{1}$ denoting the channel between $S_{1}$ and $R$, and $\alpha_{2}$ denoting the channel between $S_{2}$ and $R$. We assume that $S_{1}$, $S_{2}$ and $R$ are all equipped with one antenna only. The DF protocol is used at the relay. The DF two-way relaying strategy consists of two equal time slots.

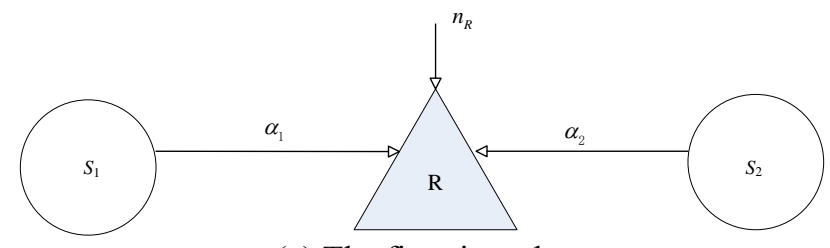

(a) The first time slot

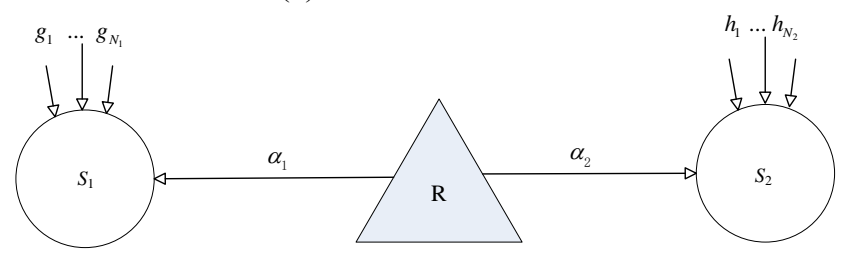

(b) The second time slot

Fig. 1. The two-way relaying system model with co-channel interference

In the first time slot, $S_{1}$ and $S_{2}$ send their messages, $x_{1}$ and $x_{2}$, to $R$, and $R$ has the following received signal:

$$
y_{R}=\alpha_{1} x_{1}+\alpha_{2} x_{2}+n_{R}
$$

where $n_{\mathrm{R}}$ is the complex Gaussian noise with zero mean and variance of $\sigma_{R}^{2}$. It is assumed that 
$\alpha_{1}$ and $\alpha_{2}$ are independent Nakagami- $m$ fading channels, respectively having fading parameter $m_{1}$ and $m_{2}$ and satisfying $\Omega_{1}=E\left\{\left|\alpha_{1}\right|^{2}\right\}$ and $\Omega_{2}=E\left\{\left|\alpha_{2}\right|^{2}\right\}$. We also denote the transmit power at $S_{1}$ and $S_{2}$ by $P_{1}=E\left\{\left|x_{1}\right|^{2}\right\}$ and $P_{2}=E\left\{\left|x_{2}\right|^{2}\right\}$, respectively.

In the second time slot, both $x_{1}$ and $x_{2}$ are decoded successfully. The relay $R$ combines the decoded symbols using physical layer network coding, obtains $x_{\mathrm{R}}$ and broadcasts it to $S_{1}$ and $S_{2}$ [31]. Therefore, after two time slots, $S_{1}$ obtains

$$
y_{1}=\alpha_{1} x_{R}+\sum_{i=1}^{N_{1}} g_{i} s_{1}^{(i)}+n_{1}
$$

where the contribution from $N_{1}$ interference signals $\left\{s_{1}^{(i)}\right\}_{i=1}^{N_{1}}$ is considered. The channels between the interferers and $S_{1}$ are $\left\{g_{i}\right\}$. They are assumed to be independent Nakagami- $m$ fading satisfying $E\left\{\left|g_{i}\right|^{2}\right\}=\Omega_{i I}$ and have fading parameter $m_{i I}$, for $i=1,2, \ldots, N_{1}$. The received signal by $S_{2}$ is

$$
y_{2}=\alpha_{2} x_{R}+\sum_{j=1}^{N_{2}} h_{j} s_{2}^{(j)}+n_{2}
$$

where $\left\{s_{2}^{(j)}\right\}_{j=1}^{N_{2}}$ are the interference signals. The channels between the interferers and $S_{2}$ are $\left\{h_{j}\right\}$. They are independent Nakagami- $m$ fading with $E\left\{\left|h_{j}\right|^{2}\right\}=\Omega_{j I}$ and have fading parameter $m_{j l}$, for $j=1,2, \ldots, N_{2}$. Furthermore, we assume that the influence of the interference at $S_{1}$ and $S_{2}$ are dominant and therefore the effect of $n_{1}$ and $n_{2}$ are neglected. The transmit power of $R$ is given by $P_{R}=E\left\{\left|x_{R}\right|^{2}\right\}$. The interference power at $S_{1}$ and $S_{2}$ are $P_{1}^{i}=E\left\{\left|s_{1}^{(i)}\right|^{2}\right\}$ and $P_{2}^{j}=E\left\{\left|s_{2}^{(j)}\right|^{2}\right\}$.

We define the following signal-to-noise ratios (SNR):

$$
\begin{gathered}
\text { SNR at } R \text { from } S_{1}: \gamma_{S_{1} R}=\frac{P_{1}\left|\alpha_{1}\right|^{2}}{\sigma_{R}^{2}} \\
\text { SNR at } R \text { from } S_{2}: \gamma_{S_{2} R}=\frac{P_{2}\left|\alpha_{2}\right|^{2}}{\sigma_{R}^{2}} \\
\text { The sum-SNR at } R: \gamma_{M A C}=\frac{P_{1}\left|\alpha_{1}\right|^{2}+P_{2}\left|\alpha_{2}\right|^{2}}{\sigma_{R}^{2}}
\end{gathered}
$$

We also define the signal-to-interference ratios:

$$
\begin{gathered}
\text { Signal-to-interference ratio at } S_{1}: \gamma_{R S_{1}}=\frac{P_{R}\left|\alpha_{1}\right|^{2}}{|\bar{g}|^{2}} \\
\text { Signal-to-interference ratio at } S_{2}: \gamma_{R S_{2}}=\frac{P_{R}\left|\alpha_{2}\right|^{2}}{|\bar{h}|^{2}}
\end{gathered}
$$

where $|\bar{g}|^{2}=\sum_{i=1}^{N_{1}}\left|g_{i}\right|^{2} P_{1}^{i}$ and $|\bar{h}|^{2}=\sum_{j=1}^{N_{2}}\left|h_{j}\right|^{2} P_{2}^{j}$ 


\section{Outage Probability Analysis}

In this section, we investigate the outage probability for the two-way relaying network. Firstly we denote the transmission rates from $S_{1}$ to $S_{2}$ and from $S_{2}$ to $S_{1}$ by $R_{1}$ and $R_{2}$, respectively. According to the rate region for the two-way relaying network in [32], an outage event exactly occurred when

$$
\begin{array}{ll}
R_{1}>\min \left\{\frac{1}{2} \log \left(1+\gamma_{S_{1} R}\right), \frac{1}{2} \log \left(1+\gamma_{R S_{2}}\right)\right\} & \text { or } \\
R_{2}>\min \left\{\frac{1}{2} \log \left(1+\gamma_{S_{2} R}\right), \frac{1}{2} \log \left(1+\gamma_{R S_{1}}\right)\right\} & \text { or } \\
R_{1}+R_{2}>\frac{1}{2} \log \left(1+\gamma_{M A C}\right) &
\end{array}
$$

Our main result is given in the following conclusion.

Conclusion: Assuming that $\bar{\gamma}_{1}=\frac{P_{1} \Omega_{1}}{\sigma_{R}^{2}}, \bar{\gamma}_{2}=\frac{P_{2} \Omega_{2}}{\sigma_{R}^{2}}, \overline{\gamma_{I 1}}=\frac{P_{1}}{P_{R} \sigma_{R}^{2}} P_{I 1} \Omega_{I 1}, \bar{\gamma}_{I 2}=\frac{P_{2}}{P_{R} \sigma_{R}^{2}} P_{I 2} \Omega_{I 2}$, $\Omega_{I 1}=\sum_{i=1}^{N_{1}} \Omega_{i I}, m_{I 1}=\sum_{i=1}^{N_{1}} m_{i I}, \Omega_{I 2}=\sum_{j=1}^{N_{2}} \Omega_{j I}, m_{I 2}=\sum_{j=1}^{N_{2}} m_{j I}, \lambda=\frac{C(\beta)}{C(1-\beta)}, \lambda_{1}=\frac{C(1)}{C(1-\beta)}$, $\lambda_{2}=\frac{C(1)}{C(\beta)}$.If the interferers have similar power at $S_{1}$ and $S_{2}$, i.e., $P_{1}^{i}=P_{1}^{j}=P_{I 1}$ and $P_{2}^{i}=P_{2}^{j}=P_{I 2}, \quad \forall i \neq j, \quad$ the outage probability $P_{\text {out }}$ is given by

$$
\begin{aligned}
& P_{\text {out }}=1-\psi+e^{-d_{1}} \sum_{p=0}^{m_{2}-1} \frac{1}{p !}\left(\frac{m_{2}}{\gamma_{2}}\right)^{p}\left\{e^{-\frac{m_{11} \lambda}{\gamma_{11}}} \delta\left[(C(\beta))^{s} F_{1}(\lambda)-F_{4}(\lambda)\right]\right. \\
& -e^{-\frac{m_{I 2}\left(\lambda_{2}-1\right)}{\gamma_{I 2}}}\left[\frac{m_{I 2}^{m_{I 2}}}{\Gamma\left(m_{I 2}\right) \gamma_{I 2}^{-m_{I 2}}} \sum_{l=0}^{m_{1}-1} \frac{1}{l !}\left(\frac{m_{1}}{\gamma_{1}}\right)^{l}\left(-F_{5}\left(\lambda_{2}-1\right)+F_{7}\left(\lambda_{2}-1\right)\right)+\delta\left(-(C(\beta))^{s} F_{2}\left(\lambda_{2}-1\right)+F_{6}\left(\lambda_{2}-1\right)\right)\right] \\
& +e^{-\frac{m_{I 1} \lambda}{\gamma_{11}}-\frac{m_{l 2}\left(\lambda_{2}-1\right)}{\gamma_{I 2}}}\left[\frac{m_{l 2}^{m_{l 2}}}{\Gamma\left(m_{I 2}\right) \gamma_{I 2}} \sum_{l=0}^{m_{1}-1} \frac{1}{l !}\left(\frac{m_{1}}{\bar{\gamma}_{1}}\right)^{l}\left(-F_{1}(\lambda) F_{5}\left(\lambda_{2}-1\right)+F_{1}(\lambda) F_{7}\left(\lambda_{2}-1\right)+F_{10}(\lambda)-F_{11}(\lambda)\right)\right. \\
& \left.\left.+\delta\left(-(C(\beta))^{s} F_{1}(\lambda) F_{2}\left(\lambda_{2}-1\right)+F_{1}(\lambda) F_{6}\left(\lambda_{2}-1\right)-F_{8}(\lambda)+F_{9}(\lambda)\right)\right]\right\} \\
& +e^{-d_{2}} \sum_{p=0}^{m_{2}-1} \frac{1}{p !}\left(\frac{m_{2}}{\bar{\gamma}_{2}}\right)^{p}\left\{e ^ { - \frac { m _ { l 2 } \lambda ^ { - 1 } } { \gamma _ { I 2 } } } \left[\sum_{l=0}^{m_{1}-1} \frac{1}{l !}\left(\frac{m_{1}}{\bar{\gamma}_{1}}\right)^{l}\left((C(1)-C(1-\beta))^{l} C(1-\beta)^{p} F_{2}\left(\lambda^{-1}\right)-F_{5}\left(\lambda^{-1}\right)\right)\right.\right. \\
& \left.+\delta\left(-(C(1)-C(1-\beta))^{s} F_{2}\left(\lambda^{-1}\right)+F_{6}\left(\lambda^{-1}\right)\right)\right] \\
& -e^{-\frac{m_{l 1}\left(\lambda_{1}-1\right)}{\gamma_{11}}}\left[\sum_{l=0}^{m_{1}-1} \frac{1}{l !}\left(\frac{m_{1}}{\gamma_{1}}\right)^{l}\left(-(C(1)-C(1-\beta))^{l} C(1-\beta)^{p} F_{1}\left(\lambda_{1}-1\right)+F_{3}\left(\lambda_{1}-1\right)\right)\right. \\
& +\delta\left[(C(1)-C(1-\beta))^{s} F_{1}\left(\lambda_{1}-1\right)-F_{4}\left(\lambda_{1}-1\right)\right] \\
& +e^{-\frac{m_{l 1}\left(\lambda_{1}-1\right)}{\gamma_{I 1}}-\frac{m_{l} \lambda^{-1}}{\gamma_{I 2}}} \sum_{l=0}^{m_{1}-1} \frac{1}{l !}\left(\frac{m_{1}}{\gamma_{1}}\right)^{l}\left[-(C(1)-C(1-\beta))^{l} C(1-\beta)^{p} F_{1}\left(\lambda_{1}-1\right) F_{2}\left(\lambda^{-1}\right)+F_{3}\left(\lambda_{1}-1\right) F_{2}\left(\lambda^{-1}\right)\right. \\
& \left.+F_{1}\left(\lambda_{1}-1\right) F_{12}\left(\lambda^{-1}\right)-F_{10}\left(\lambda_{1}-1\right)-F_{13}\left(\lambda_{1}-1\right)+F_{11}\left(\lambda_{1}-1\right)\right] \\
& \left.+\delta\left[(C(1)-C(\beta))^{s} F_{1}\left(\lambda_{1}-1\right) F_{2}\left(\lambda^{-1}\right)-F_{1}\left(\lambda_{1}-1\right) F_{14}\left(\lambda^{-1}\right)+F_{8}\left(\lambda_{1}-1\right)-F_{9}\left(\lambda_{1}-1\right)\right]\right\}
\end{aligned}
$$


In (10), the parameters are

$$
\begin{aligned}
& \psi=\frac{\Gamma\left(m_{1}, m_{1}(C(1)-C(1-\beta)) / \bar{\gamma}_{1}\right)}{\Gamma\left(m_{1}\right)} \frac{\Gamma\left(m_{2}, m_{2} C(1-\beta) / \bar{\gamma}_{2}\right)}{\Gamma\left(m_{2}\right)} \\
& -\exp \left(-\frac{m_{2} C(1)}{\bar{\gamma}_{2}}\right) \frac{m_{1}^{m_{1}}}{\Gamma\left(m_{1}\right) \bar{\gamma}_{1}} \sum_{p=0}^{m_{2}-1} \frac{m_{2}^{p}}{p ! \bar{\gamma}_{2}^{p}} \sum_{j=0}^{p} \frac{\left(\begin{array}{c}
p \\
j
\end{array}\right)(-1)^{j} C(1)^{p-j}}{\left(\frac{m_{1}}{\bar{\gamma}_{1}}-\frac{m_{2}}{\bar{\gamma}_{2}}\right)^{p+m_{1}}} \Gamma\left(j+m_{1},\left(\frac{m_{1}}{\bar{\gamma}_{1}}-\frac{m_{2}}{\bar{\gamma}_{2}}\right)(C(1)-C(1-\beta))\right), \\
& +\exp \left(-\frac{m_{2} C(1)}{\bar{\gamma}_{2}}\right) \frac{m_{1}^{m_{1}}}{\Gamma\left(m_{1}\right) \gamma_{1}^{-m_{1}}} \sum_{p=0}^{m_{2}-1} \frac{m_{2}^{p}}{p ! \bar{\gamma}_{2}^{p}} \sum_{j=0}^{p} \frac{\left(\begin{array}{c}
p \\
j
\end{array}\right)(-1)^{j} C(1)^{p-j}}{\left(\frac{m_{1}}{\bar{\gamma}_{1}}-\frac{m_{2}}{\bar{\gamma}_{2}}\right)^{p+m_{1}}} \Gamma\left(j+m_{1},\left(\frac{m_{1}}{\bar{\gamma}_{1}}-\frac{m_{2}}{\bar{\gamma}_{2}}\right) C(\beta)\right) \\
& d_{1}=\frac{m_{1} C(\beta)}{\gamma_{1}}+\frac{m_{2}(C(1)-C(\beta))}{\overline{\gamma_{2}}}, d_{2}=\frac{m_{2} C(1-\beta)}{\bar{\gamma}_{2}}+\frac{m_{1}(C(1)-C(1-\beta))}{\overline{\gamma_{1}}}, \\
& \delta=\frac{m_{1}^{m_{1}}}{\Gamma\left(m_{1}\right) \bar{\gamma}_{1}} \sum_{j=0}^{p} \frac{\left(\begin{array}{l}
p \\
j
\end{array}\right)(-1)^{j} C(1)^{p-j} \Gamma\left(j+m_{1}\right)^{j+m_{1}-1}}{\left(\frac{m_{1}}{\bar{\gamma}_{1}}-\frac{m_{2}}{\bar{\gamma}_{2}}\right)^{j+m_{1}}} \sum_{s=0} \frac{1}{s !}\left(\frac{m_{1}}{\bar{\gamma}_{1}}-\frac{m_{2}}{\bar{\gamma}_{2}}\right)^{s}, \\
& F_{1}(x)=\sum_{f=0}^{m_{I 1}-1} \frac{1}{f !}\left(\frac{m_{I 1} x}{\bar{\gamma}_{I 1}}\right)^{f}, F_{2}(x)=\sum_{h=0}^{m_{I 2}-1} \frac{1}{h !}\left(\frac{m_{I 2} x}{\bar{\gamma}_{I 2}}\right)^{h}, \\
& F_{3}(x)=\frac{m_{I 1}^{m_{I 1}} C(1-\beta)^{l+p}}{\Gamma\left(m_{I 1}\right) \bar{\gamma}_{I 1}^{-m_{I 1}}} \frac{\Gamma\left(l+m_{I 1}\right)}{\left(\frac{m_{I 1}}{\bar{\gamma}_{I 1}}+\frac{m_{1}}{\bar{\gamma}_{1}} C(1-\beta)\right)^{l+m_{l 1}}} \sum_{i=0}^{l+m_{I 1}-1} \frac{1}{i !}\left(\frac{m_{I 1}}{\bar{\gamma}_{I 1}}+\frac{m_{1}}{\bar{\gamma}_{1}} C(1-\beta)\right)^{i} x^{i}, \\
& F_{4}(x)=\frac{m_{I 1}^{m_{I 1}} C(1-\beta)^{s}}{\Gamma\left(m_{I 1}\right) \bar{\gamma}_{I 1}^{-m_{I 1}}} \frac{\Gamma\left(s+m_{I 1}\right)}{\left(\frac{m_{I 1}}{\bar{\gamma}_{I 1}}+\left(\frac{m_{1}}{\bar{\gamma}_{1}}-\frac{m_{2}}{\bar{\gamma}_{2}}\right) C(1-\beta)\right)^{s+m_{I 1}}} \sum_{n=0}^{s+m_{l 1}-1} \frac{1}{n !}\left(\frac{m_{I 1}}{\bar{\gamma}_{I 1}}+\left(\frac{m_{1}}{\bar{\gamma}_{1}}-\frac{m_{2}}{\bar{\gamma}_{2}}\right) C(1-\beta)\right)^{n} x^{n}, \\
& \left.F_{5}(x)=\frac{m_{I 2}^{m_{I 2}}}{\Gamma\left(m_{I 2}\right) \gamma_{I 2}^{-m_{I 2}}} \sum_{i=0}^{l} \frac{\left(\begin{array}{l}
l \\
i
\end{array}\right)(-1)^{i} C(1)^{l-i} C(\beta)^{i} \Gamma\left(i+p+m_{I 2}\right)_{i+p+m_{l 2}-1}}{\left(\frac{m_{I 2}}{\bar{\gamma}_{I 2}}+\left(\frac{m_{1}}{\bar{\gamma}_{1}}-\frac{m_{2}}{\bar{\gamma}_{2}}\right) C(\beta)\right)^{i+p+m_{I 2}}} \sum_{t=0}^{t !} \frac{m_{I 2}}{\bar{\gamma}_{I 2}}+\left(\frac{m_{1}}{\bar{\gamma}_{1}}-\frac{m_{2}}{\bar{\gamma}_{2}}\right) C(\beta)\right)^{t} x^{t}, \\
& F_{6}(x) \frac{m_{I 2}^{m_{I 2}}}{\Gamma\left(m_{I 2}\right) \bar{\gamma}_{I 2}^{-m_{I 2}}} \sum_{n=0}^{s} \frac{\left(\begin{array}{c}
s \\
n
\end{array}\right)(-1)^{n} C(1)^{s-n} C(\beta)^{n}}{\left(\frac{m_{I 2}}{\bar{\gamma}_{I 2}}+\left(\frac{m_{1}}{\bar{\gamma}_{1}}-\frac{m_{2}}{\bar{\gamma}_{2}}\right) C(\beta)\right)^{n+m_{I 2}}} \sum_{k=0}^{n+m_{I 2}-1} \frac{1}{k !}\left(\frac{m_{I 2}}{\bar{\gamma}_{I 2}}+\left(\frac{m_{1}}{\bar{\gamma}_{1}}-\frac{m_{2}}{\bar{\gamma}_{2}}\right) C(\beta)\right)^{k} x^{k},
\end{aligned}
$$

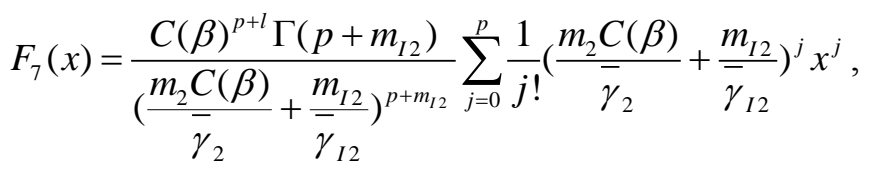




$$
\begin{aligned}
& \left.F_{8}(x) \frac{m_{I 1}^{m_{I 1}}}{\Gamma\left(m_{I 1}\right) \bar{\gamma}_{I 1}^{m_{I 1}}} \frac{m_{I 2}^{m_{I 2}}}{\Gamma\left(m_{I 2}\right) \bar{\gamma}_{I 2}} \sum_{n=0}^{s} \frac{\left(\begin{array}{l}
s \\
n
\end{array}\right)(-1)^{n} C(1)^{s-n} C(\beta)^{n} \Gamma\left(n+m_{I 2}\right)_{n+m_{I 2}-1}}{\left(\frac{m_{I 2}}{\bar{\gamma}_{I 2}}-\frac{m_{1}}{\bar{\gamma}_{1}}+\frac{m_{2}}{\bar{\gamma}_{2}}\right)^{n+m_{I 2}}} \sum_{k=0}^{\frac{m_{I 2}}{k !}}-\frac{m_{1}}{\bar{\gamma}_{1}}+\frac{m_{2}}{\bar{\gamma}_{2}}\right)^{k} \\
& \sum_{a=0}^{k} \frac{\left(\begin{array}{c}
k \\
a
\end{array}\right)(-1)^{a} \lambda_{2}^{k-a} \lambda^{-a} \Gamma\left(a+m_{I 1}\right)}{\left(\frac{m_{I 1}}{\bar{\gamma}_{I 1}}-\frac{m_{I 2}}{\bar{\gamma}_{I 2} \lambda}+\frac{m_{1}}{\bar{\gamma}_{1} \lambda}-\frac{m_{2}}{\bar{\gamma}_{2} \lambda}\right)^{a+m_{I 1}}} \sum_{b=0}^{a} \frac{1}{b !}\left(\frac{m_{I 1}}{\bar{\gamma}_{I 1}}-\frac{m_{I 2}}{\bar{\gamma}_{I 2} \lambda}+\frac{m_{1}}{\bar{\gamma}_{1} \lambda}-\frac{m_{2}}{\bar{\gamma}_{2} \lambda}\right)^{b} x^{b} \\
& F_{9}(x) \frac{m_{I 1}^{m_{I 1}}}{\Gamma\left(m_{I 1}\right)_{I 1}^{-m_{I 1}}} \sum_{h=0}^{m_{I 2}-1} \frac{1}{h !}\left(\frac{m_{I 2}}{\bar{\gamma}_{I 2}}\right)^{h} \sum_{b=0}^{h} \frac{\left(\begin{array}{l}
h \\
b
\end{array}\right) \lambda_{2}^{h-b}\left(-\lambda^{-1}\right)^{b} \Gamma\left(s+b+m_{I 1}\right)}{\left(\frac{m_{I 1}}{\bar{\gamma}_{I 1}}-\frac{m_{I 2}}{\bar{\gamma}_{I 2} \lambda}+\left(\frac{m_{1}}{\bar{\gamma}_{1}}-\frac{m_{2}}{\bar{\gamma}_{2}}\right) C(1-\beta)\right)^{s+p+m_{I 1}}}, \\
& \sum_{q=0}^{s+b+m_{I 1}-1} \frac{1}{q !}\left(\frac{m_{I 1}}{\bar{\gamma}_{I 1}}-\frac{m_{I 2}}{\bar{\gamma}_{I 2} \lambda}+\left(\frac{m_{1}}{\bar{\gamma}_{1}}-\frac{m_{2}}{\bar{\gamma}_{2}}\right) C(1-\beta)\right)^{q} \lambda^{q} \\
& \left.F_{10}(x)=\sum_{i=0}^{l} \frac{\left(\begin{array}{l}
l \\
i
\end{array}\right)(-1)^{i} C(1)^{l-i} C(\beta)^{i} \Gamma\left(i+p+m_{I 2}\right)_{i+p+m_{I 2}-1}}{\left(\frac{m_{I 2}}{\gamma_{I 2}}+\left(\frac{m_{1}}{\bar{\gamma}_{1}}-\frac{m_{2}}{\bar{\gamma}_{2}}\right) C(\beta)\right)^{i+p+m_{I 2}}} \sum_{c=0}^{c !} \frac{m_{I 2}}{\bar{\gamma}_{I 2}}+\left(\frac{m_{1}}{\bar{\gamma}_{1}}-\frac{m_{2}}{\bar{\gamma}_{2}}\right) C(\beta)\right)^{c} \\
& \sum_{d=0}^{c} \frac{\left(\begin{array}{c}
c \\
d
\end{array}\right) \lambda_{2}^{c-d}\left(-\lambda^{-1}\right)^{d} \Gamma\left(d+m_{I 1}\right)}{\left(\frac{m_{I 1}}{\bar{\gamma}_{I 1}}-\frac{m_{I 2}}{\bar{\gamma}_{I 2} \lambda}+\left(-\frac{m_{1}}{\bar{\gamma}_{1}}+\frac{m_{2}}{\bar{\gamma}_{2}}\right) C(1-\beta)\right)^{d+m_{I 1}}} \sum_{q=0}^{d+m_{I}-1} \frac{1}{q !}\left(\frac{m_{I 1}}{\bar{\gamma}_{I 1}}-\frac{m_{I 2}}{\bar{\gamma}_{I 2} \lambda}+\left(-\frac{m_{1}}{\bar{\gamma}_{1}}+\frac{m_{2}}{\bar{\gamma}_{2}}\right) C(1-\beta)\right)^{q} x^{q} \\
& F_{11}(x)=\frac{m_{I 1}^{m_{I 1}} C(1-\beta)^{l} C(\beta)^{p}}{\Gamma\left(m_{I 1}\right) \bar{\gamma}_{I 1}^{m_{I 1}}} \frac{\Gamma\left(p+m_{I 2}\right)}{\left(\frac{m_{I 2}}{\bar{\gamma}_{I 2}}+\frac{m_{2}}{\bar{\gamma}_{2}} C(\beta)\right)^{p+m_{I 2}}} \sum_{j=0}^{p+m_{I 2}-1} \frac{1}{j !}\left(\frac{m_{I 2}}{\bar{\gamma}_{I 2}}+\frac{m_{2}}{\bar{\gamma}_{2}} C(\beta)\right)^{j} \\
& \sum_{s=0}^{j} \frac{\left.\left(\begin{array}{c}
s \\
j
\end{array}\right)(-1)^{s} \lambda_{2}^{s-j} \lambda^{-1}\right)^{s} \Gamma\left(l+s+m_{I 1}\right)}{\left(\frac{m_{I 1}}{\bar{\gamma}_{I 1}}-\frac{m_{I 2}}{\bar{\gamma}_{I 2} \lambda}+\left(\frac{m_{1}}{\bar{\gamma}_{1}}-\frac{m_{2}}{\bar{\gamma}_{2}}\right) C(1-\beta)\right)^{l+s+m_{I 1}}} \sum_{n=0}^{l+s+m_{11}-1} \frac{1}{n !}\left(\frac{m_{I 1}}{\gamma_{I 1}}-\frac{m_{I 2}}{\bar{\gamma}_{I 2} \lambda}+\left(\frac{m_{1}}{\bar{\gamma}_{1}}-\frac{m_{2}}{\bar{\gamma}_{2}}\right) C(1-\beta)\right)^{n} x^{n} \\
& \left.F_{12}(x)=\frac{m_{I 2}^{m_{I 2}}}{\Gamma\left(m_{I 2}\right) \bar{\gamma}_{I 2}} \sum_{i=0}^{l} \frac{\left(\begin{array}{l}
l \\
i
\end{array}\right)(-1)^{i} C(1)^{l-i} C(\beta)^{i} \Gamma\left(i+p+m_{I 2}\right)_{i+p+m_{l 2}-1}}{\left(\frac{m_{I 2}}{\gamma_{I 2}}+\left(\frac{m_{1}}{\bar{\gamma}_{1}}-\frac{m_{2}}{\gamma_{2}}\right) C(\beta)\right)^{i+p+m_{I 2}}} \sum_{t=0}^{t !} \frac{m_{I 2}}{\bar{\gamma}_{I 2}}+\left(\frac{m_{1}}{\gamma_{1}}-\frac{m_{2}}{\bar{\gamma}_{2}}\right) C(\beta)\right)^{t} x^{t},
\end{aligned}
$$




$$
\begin{aligned}
& \left.F_{13}(x)=\frac{m_{I 2}^{m_{I 2}}}{\Gamma\left(m_{I 2}\right) \bar{\gamma}_{I 2}^{-m_{I 2}}} \sum_{i=0}^{l} \frac{\left(\begin{array}{l}
l \\
i
\end{array}\right)(-1)^{i} C(1)^{l-i} C(\beta)^{i} \Gamma\left(i+p+m_{I 2}\right)_{i+p+m_{12}-1}}{\left(\frac{m_{I 2}}{\bar{\gamma}_{I 2}}+\left(\frac{m_{1}}{\bar{\gamma}_{1}}-\frac{m_{2}}{\bar{\gamma}_{2}}\right) C(\beta)\right)^{i+p+m_{I 2}}} \sum_{c=0}^{c !} \frac{m_{I 2}}{\bar{\gamma}_{I 2}}+\left(\frac{m_{1}}{\bar{\gamma}_{1}}-\frac{m_{2}}{\bar{\gamma}_{2}}\right) C(\beta)\right)^{c} \\
& \sum_{d=0}^{c} \frac{\left(\begin{array}{c}
c \\
d
\end{array}\right) \lambda_{2}^{c-d}\left(-\lambda^{-1}\right)^{d} \Gamma\left(d+m_{I 1}\right)}{\left(\frac{m_{I 1}}{\bar{\gamma}_{I 1}}-\frac{m_{I 2}}{\bar{\gamma}_{I 2} \lambda}+\left(-\frac{m_{1}}{\bar{\gamma}_{1}}+\frac{m_{2}}{\bar{\gamma}_{2}}\right) C(1-\beta)\right)^{d+m_{I 1}}} \sum_{q=0}^{d+m_{I}-1} \frac{1}{q !}\left(\frac{m_{I 1}}{\bar{\gamma}_{I 1}}-\frac{m_{I 2}}{\bar{\gamma}_{I 2} \lambda}+\left(-\frac{m_{1}}{\bar{\gamma}_{1}}+\frac{m_{2}}{\bar{\gamma}_{2}}\right) C(1-\beta)\right)^{q} x^{q} \\
& F_{14}(x)=\frac{m_{I 2}^{m_{I 2}}}{\Gamma\left(m_{I 2}\right) \bar{\gamma}_{I 2}^{-m_{I 2}}} \sum_{n=0}^{s} \frac{\left(\begin{array}{l}
s \\
n
\end{array}\right)(-1)^{n} C(1)^{s-n} C(\beta)^{n} \Gamma\left(n+m_{I 1}\right)_{n+m_{I 1}-1}}{\left(\frac{m_{I 2}}{\bar{\gamma}_{I 2}}+\left(-\frac{m_{1}}{\bar{\gamma}_{1}}+\frac{m_{2}}{\bar{\gamma}_{2}}\right) C(\beta)\right)^{n+m_{I 1}}} \sum_{k=0}^{\frac{1}{k !}}\left(\frac{m_{I 2}}{\bar{\gamma}_{I 2}}+\left(-\frac{m_{1}}{\bar{\gamma}_{1}}+\frac{m_{2}}{\bar{\gamma}_{2}}\right) C(\beta)\right)^{k} x^{k}
\end{aligned}
$$

Proof: See Appendix.

\section{Numerical Results}

Here, we present the numerical result to validate the analytical result of the outage probability. Fig. 2 portrays the outage probability of DF two-way relaying network for different $R_{2}$ and by setting $P_{1}=P_{2}, m_{1}=2, \Omega_{1}=1.5, m_{2}=2$, and $\Omega_{2}=2$. In the simulation, we set the transmission rates by $R_{1}=0.7, P_{1}^{i}=P_{1}^{j}=P_{I 1}=1, P_{2}^{i}=P_{2}^{j}=P_{I 2}=1,\left\{m_{i l}\right\}_{i=1}^{2}=\{2,2\},\left\{\Omega_{i l}\right\}_{i=1}^{2}=\{1.8,2\}$, $\left\{m_{j l}\right\}_{j=1}^{2}=\{2,2\},\left\{\Omega_{j l}\right\}_{j=1}^{2}=\{1.8,2\}, P_{\mathrm{R}}=30 \mathrm{~dB}, N_{1}=N_{2}=2$. It is clearly observed that the approximate result (10) matches the Monte Carlo result perfectly.

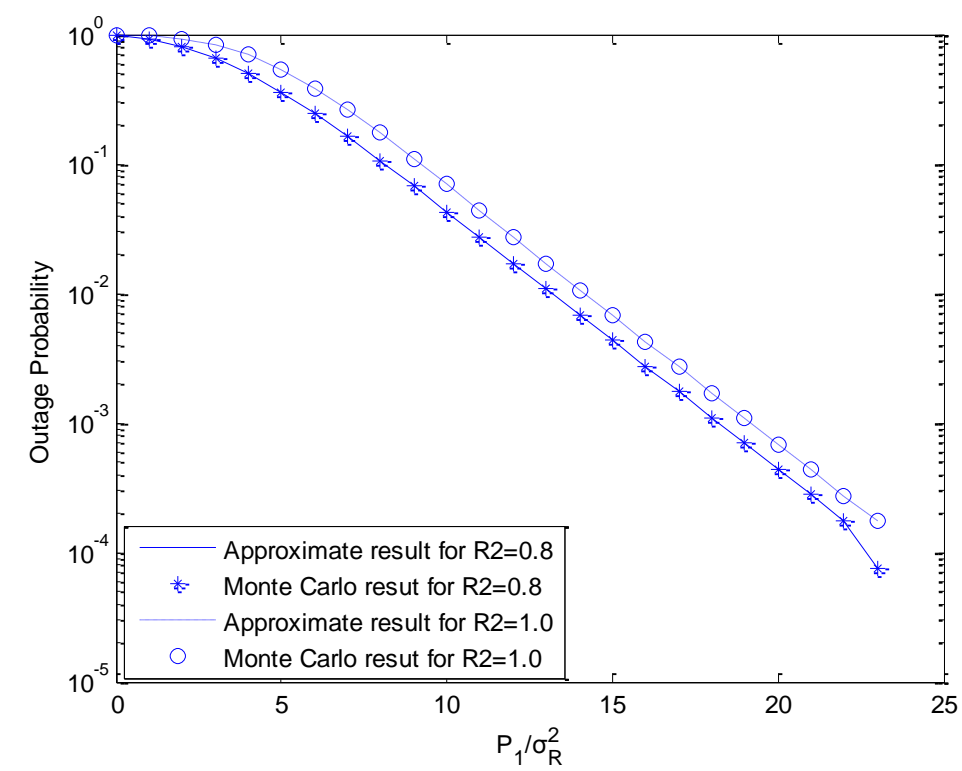

Fig. 2. Outage probability of DF two-way relaying system for different $R_{2}$ 


\section{Conclusion}

Assuming a two-way DF relaying system with Nakagami- $m$ fading multiple co-channel interferers at the source/terminal nodes and a noisy relay, an approximate expression for the outage probability was derived. In all the comparisons, an excellent match between the approximate and simulation result has been observed.

\section{APPENDIX}

\section{Proof}

We define the function $C(x) \stackrel{\Delta}{=} 2^{2 x R}-1$ with $R=R_{1}+R_{2}$. Then, (9) is recast as

$$
\left\{\gamma_{S_{1} R}<C(\beta) \text { or } \gamma_{R S_{1}}<C(1-\beta) \text { or } \gamma_{S_{2} R}<C(1-\beta) \text { or } \gamma_{R S_{2}}<C(\beta) \text { or } \gamma_{M A C}<C(1)\right\}
$$

where $\beta=R_{1} / R$, for $0 \leq \beta \leq 1$. Therefore, the outage probability is given by

$P_{\text {out }}=P\left\{Z_{1}<C(\beta)\right.$ or $Z_{1}<\beta_{1} C(1-\beta)$ or $Z_{2}<C(1-\beta)$ or $Z_{2}<\beta_{2} C(\beta)$ or $\left.Z_{1}+Z_{2}<C(1)\right\}(13)$

where $\beta_{1}=\left.\frac{P_{1}}{P_{R} \sigma_{R}^{2}}||^{-}\right|^{2}, \beta_{2}=\frac{P_{2}}{P_{R} \sigma_{R}^{2}}|h|^{2}, Z_{1}=\frac{P_{1}\left|\alpha_{1}\right|^{2}}{\sigma_{R}^{2}}$ and $Z_{2}=\frac{P_{2}\left|\alpha_{2}\right|^{2}}{\sigma_{R}^{2}}$. From (13), we see

that $P_{\text {out }}$ is affected by the cumulative density functions (CDFs) of $Z_{1}, Z_{2}, \beta_{1}$ and $\beta_{2}$. It is easily derived that

$$
C(\beta)+C(1-\beta) \leq C(1) \quad 0 \leq \beta \leq 1
$$

Therefore, we obtain the expressions of $P_{\text {out }}$ for different cases:

$$
\begin{aligned}
& P_{\text {out }}^{(1)}=P\left\{Z_{1}<C(\beta) \text { or } Z_{2}<C(1-\beta) \text { or } Z_{1}+Z_{2}<C(1)\right\} \quad \beta_{1} \leq \frac{C(\beta)}{C(1-\beta)}, \beta_{2} \leq \frac{C(1-\beta)}{C(\beta)} \\
& P_{\text {out }}^{(2)}=P\left\{Z_{1}<C(\beta) \text { or } Z_{2}<\beta_{2} C(\beta) \text { or } Z_{1}+Z_{2}<C(1)\right\} \\
& \beta_{1} \leq \frac{C(\beta)}{C(1-\beta)}, \frac{C(1-\beta)}{C(\beta)}<\beta_{2} \leq \frac{C(1)}{C(\beta)}-1 \\
& P_{\text {out }}^{(3)}=P\left\{Z_{1}<C(\beta) \text { or } Z_{2}<\beta_{2} C(\beta)\right\} \quad \beta_{1} \leq \frac{C(\beta)}{C(1-\beta)}, \beta_{2}>\frac{C(1)}{C(\beta)}-1 \\
& P_{\text {out }}^{(4)}=P\left\{Z_{1}<\beta_{1} C(1-\beta) \text { or } Z_{2}<C(1-\beta) \text { or } Z_{1}+Z_{2}<C(1)\right\} \\
& \frac{C(\beta)}{C(1-\beta)}<\beta_{1} \leq \frac{C(1)}{C(1-\beta)}-1, \beta_{2} \leq \frac{C(1-\beta)}{C(\beta)} \\
& P_{\text {out }}^{(5)}=P\left\{Z_{1}<\beta_{1} C(1-\beta) \text { or } Z_{2}<C(1-\beta)\right\} \quad \beta_{1}>\frac{C(1)}{C(1-\beta)}-1, \beta_{2} \leq \frac{C(1-\beta)}{C(\beta)} \\
& P_{\text {out }}^{(6)}=P\left\{Z_{1}<\beta_{1} C(1-\beta) \text { or } Z_{2}<\beta_{2} C(\beta) \text { or } Z_{1}+Z_{2}<C(1)\right\} \\
& \beta_{1}>\frac{C(1)}{C(1-\beta)}-1, \beta_{2}>\frac{C(1-\beta)}{C(\beta)}, \beta_{1} C(1-\beta)+\beta_{2} C(\beta) \leq C(1)
\end{aligned}
$$




$$
\begin{aligned}
& P_{\text {out }}^{(7)}=P\left\{Z_{1}<\beta_{1} C(1-\beta) \text { or } Z_{2}<\beta_{2} C(\beta)\right\} \\
& \beta_{1}>\frac{C(\beta)}{C(1-\beta)}, \beta_{2}>\frac{C(1-\beta)}{C(\beta)}, \beta_{1} C(1-\beta)+\beta_{2} C(\beta) \leq C(1)
\end{aligned}
$$

and

$$
\begin{aligned}
& P_{\text {out }}=P_{\text {out }}^{(1)} F_{\bar{\beta}_{1}}(\lambda) F_{\bar{\beta}_{2}}\left(\lambda^{-1}\right)+\int_{0}^{\lambda} \int_{\lambda^{-1}}^{\lambda_{2}-1} P_{\text {out }}^{(2)} f_{\bar{\beta}_{2}}\left(\beta_{2}\right) d \beta_{2} f_{\bar{\beta}_{1}}\left(\beta_{1}\right) d \beta_{1} \\
& +\int_{0}^{\lambda} \int_{\lambda_{2}-1}^{\infty} P_{\text {out }}^{(3)} f_{\bar{\beta}_{2}}\left(\beta_{2}\right) d \beta_{2} f_{\bar{\beta}_{1}}\left(\beta_{1}\right) d \beta_{1}+\int_{\lambda}^{\lambda_{1}-1} \int_{0}^{\lambda^{-1}} P_{\text {out }}^{(4)} f_{\bar{\beta}_{2}}\left(\beta_{2}\right) d \beta_{2} f_{\bar{\beta}_{1}}\left(\beta_{1}\right) d \beta_{1} \\
& +\int_{\lambda_{1}-1}^{\infty} \int_{0}^{\lambda^{-1}} P_{\text {out }}^{(5)} f_{\bar{\beta}_{2}}\left(\beta_{2}\right) d \beta_{2} f_{\bar{\beta}_{1}}\left(\beta_{1}\right) d \beta_{1}+\int_{\lambda}^{\lambda_{1}-1} \int_{\lambda^{-1}}^{\lambda_{2}-\frac{\beta_{1}}{\lambda}} P_{\text {out }}^{(6)} f_{\bar{\beta}_{2}}\left(\beta_{2}\right) d \beta_{2} f_{\bar{\beta}_{1}}\left(\beta_{1}\right) d \beta_{1} \\
& +\int_{\lambda}^{\infty} \int_{\lambda^{-1}}^{\infty} P_{\text {out }}^{(7)} f_{\bar{\beta}_{2}}\left(\beta_{2}\right) d \beta_{2} f_{\bar{\beta}_{1}}\left(\beta_{1}\right) d \beta_{1}-\int_{\lambda}^{\lambda_{1}-1} \int_{\lambda^{-1}}^{\lambda_{2}-\frac{\beta_{1}}{\lambda}} P_{\text {out }}^{(7)} f_{\bar{\beta}_{2}}\left(\beta_{2}\right) d \beta_{2} f_{\bar{\beta}_{1}}\left(\beta_{1}\right) d \beta_{1}
\end{aligned}
$$

where $F_{\bar{\beta}_{1}}(x), \quad F_{\bar{\beta}_{2}}(x), f_{\bar{\beta}_{1}}(x), f_{\bar{\beta}_{2}}(x)$ denote the CDFs and the probability density function (PDF)s for $\beta_{1}$ and $\beta_{2}$ respectively. With $Z_{1}=\frac{P_{1}\left|\alpha_{1}\right|^{2}}{\sigma_{R}^{2}}, Z_{2}=\frac{P_{2}\left|\alpha_{2}\right|^{2}}{\sigma_{R}^{2}}$, the CDFs of $Z_{1}$ and $Z_{2}$ are, respectively, given by

$$
F_{Z_{1}}(x)=1-\frac{\Gamma\left(m_{1}, m_{1} x / \overline{\gamma_{1}}\right)}{\Gamma\left(m_{1}\right)} \quad x \geq 0
$$

where $\bar{\gamma}_{1}=\frac{P_{1} \Omega_{1}}{\sigma_{R}^{2}}$, and

$$
F_{Z_{2}}(x)=1-\frac{\Gamma\left(m_{2}, m_{2} x / \bar{\gamma}_{2}\right)}{\Gamma\left(m_{2}\right)} \quad x \geq 0
$$

where $\bar{\gamma}_{2}=\frac{P_{2} \Omega_{2}}{\sigma_{R}^{2}}$, and the PDFs of $Z_{1}$ and $Z_{2}$ are, respectively, given by

$$
\begin{array}{cc}
f_{Z_{1}}(x)=\frac{m_{1}^{m_{1}} x^{m_{1}-1}}{\Gamma\left(m_{1}\right)} e^{-\frac{m_{1} x}{\overline{\gamma_{1}}}} & x \geq 0 \\
f_{Z_{2}}(x)=\frac{m_{2}^{m_{2}} x^{m_{2}-1}}{\Gamma\left(m_{2}\right)} e^{-\frac{m_{2} x}{\gamma_{2}}} & x \geq 0
\end{array}
$$

Following (15)-(21), we have

$$
\begin{aligned}
& P_{\text {out }}^{(1)}=G_{1}(C(1-\beta), C(\beta)) \\
& P_{\text {out }}^{(2)}=G_{1}\left(\beta_{2} C(\beta), C(\beta)\right) \\
& P_{\text {out }}^{(3)}=G_{2}\left(C(\beta), \beta_{2} C(\beta)\right) \\
& P_{\text {out }}^{(4)}=G_{1}\left(C(1-\beta), \beta_{1} C(1-\beta)\right) \\
& P_{\text {out }}^{(5)}=G_{2}\left(\beta_{1} C(1-\beta), C(1-\beta)\right) \\
& P_{\text {out }}^{(6)}=G_{1}\left(\beta_{2} C(\beta), \beta_{1} C(1-\beta)\right) \\
& P_{\text {out }}^{(7)}=G_{2}\left(\beta_{1} C(1-\beta), \beta_{2} C(\beta)\right)
\end{aligned}
$$

where 


$$
\begin{gathered}
G_{1}(x, y)=1-\psi(x, y) \\
G_{2}(x, y)=1-\frac{\Gamma\left(m_{1}, m_{1} x / \overline{\gamma_{1}}\right)}{\Gamma\left(m_{1}\right)} \frac{\Gamma\left(m_{2}, m_{2} x / \gamma_{2}\right)}{\Gamma\left(m_{2}\right)}
\end{gathered}
$$

and $\psi(x, y)$ is

$$
\begin{aligned}
& \psi(x, y)=\frac{\Gamma\left(m_{1}, m_{1}(C(1)-y) / \overline{\gamma_{1}}\right)}{\Gamma\left(m_{1}\right)} \frac{\Gamma\left(m_{2}, m_{2} y / \bar{\gamma}_{2}\right)}{\Gamma\left(m_{2}\right)} \\
& -\exp \left(-\frac{m_{2} C(1)}{\bar{\gamma}_{2}}\right) \frac{m_{1}^{m_{1}}}{\Gamma\left(m_{1}\right) \bar{\gamma}_{1}^{-m_{1}}} \sum_{p=0}^{m_{2}} \frac{m_{2}^{p}}{p ! \bar{\gamma}_{2}^{p}} \sum_{j=0}^{p} \frac{\left(\begin{array}{c}
p \\
j
\end{array}\right)(-1)^{j} C(1)^{p-j}}{\left(\frac{m_{1}}{\gamma_{1}}-\frac{m_{2}}{\gamma_{2}}\right)^{p+m_{1}}} \Gamma\left(j+m_{1},\left(\frac{m_{1}}{\gamma_{1}}-\frac{m_{2}}{\gamma_{2}}\right)(C(1)-y)\right) \\
& +\exp \left(-\frac{m_{2} C(1)}{\bar{\gamma}_{2}}\right) \frac{m_{1}^{m_{1}}}{\Gamma\left(m_{1}\right) \gamma_{1}^{-m_{1}}} \sum_{p=0}^{m_{2}-1} \frac{m_{2}^{p}}{p ! \gamma_{2}^{p}} \sum_{j=0}^{p} \frac{\left(\begin{array}{l}
p \\
j
\end{array}\right)(-1)^{j} C(1)^{p-j}}{\left(\frac{m_{1}}{\gamma_{1}}-\frac{m_{2}}{\gamma_{2}}\right)^{p+m_{1}}} \Gamma\left(j+m_{1},\left(\frac{m_{1}}{\gamma_{1}}-\frac{m_{2}}{\gamma_{2}}\right) x\right)
\end{aligned}
$$

According to the definitions $\beta_{1}=\frac{P_{1}}{P_{R} \sigma_{R}^{2}}|\bar{g}|^{2}, \beta_{2}=\frac{P_{2}}{P_{R} \sigma_{R}^{2}}|\bar{h}|^{2}$ and the CDFs of $\left|\frac{g}{\mid}\right|^{2}$ and $\left.||^{-}\right|^{2}$ in [33], the CDFs and PDFs of $\beta_{1}$ and $\beta_{2}$ are given by

$$
\begin{array}{cc}
F_{\bar{\beta}_{1}}(x)=1-\frac{\Gamma\left(m_{I 1}, m_{I 1} x / \gamma_{I 1}\right)}{\Gamma\left(m_{I 1}\right)} & x \geq 0 \\
F_{\overline{\beta_{2}}}(x)=1-\frac{\Gamma\left(m_{I 2}, m_{I 2} x / \overline{\gamma_{I 2}}\right)}{\Gamma\left(m_{I 2}\right)} & x \geq 0 \\
f_{\overline{\beta_{1}}}(x)=\frac{m_{I 1}^{m_{I 1}} x^{m_{I 1}-1}}{\Gamma\left(m_{I 1}\right)} e^{-\frac{m_{11} x}{\gamma_{I 1}}} & x \geq 0 \\
f_{\bar{\beta}_{2}}(x)=\frac{m_{I 2}^{m_{I 2}} x^{m_{I 2}-1}}{\Gamma\left(m_{I 2}\right)} e^{-\frac{m_{I 2} x}{\gamma_{I 2}}} & x \geq 0
\end{array}
$$

in which $\bar{\gamma}_{I 1}=\frac{P_{1}}{P_{R} \sigma_{R}^{2}} P_{I 1} \Omega_{I 1}, \overline{\gamma_{I 2}}=\frac{P_{2}}{P_{R} \sigma_{R}^{2}} P_{I 2} \Omega_{I 2}$. Without loss of generality, hereafter we assume that no power control is used, i.e., $P_{1}^{i}=P_{I 1}, P_{2}^{j}=P_{I 2}$. In order to render (39) and (40) an accurate approximation, the required parameters $m_{I 1}, m_{I 2}, \Omega_{I 1}$ and $\Omega_{I 2}$ must be calculated. To this end, we shall use moment-based estimators [34] for the computation of such parameters. Firstly, let $\phi_{1}=\sum_{i=1}^{N_{1}}\left|g_{i}\right|^{2}, \phi_{2}=\sum_{j=1}^{N_{2}}\left|h_{j}\right|^{2}$. Then, moment-based estimators can be written from the exact moments of $\phi_{1}$ and $\phi_{2}$, i.e., $\Omega_{I 1}=E\left\{\phi_{1}\right\}, \Omega_{I 2}=E\left\{\phi_{2}\right\}$, $m_{I 1}=\sum_{i=1}^{N_{1}} m_{i I}, m_{I 2}=\sum_{j=1}^{N_{2}} m_{j I}$, where $\Omega_{I 1}$ and $\Omega_{21}$ are easily attained as $\Omega_{I 1}=\sum_{i=1}^{N_{1}} \Omega_{i I}$ and 
$\Omega_{I 2}=\sum_{j=1}^{N_{2}} \Omega_{j I}$.Substituting (27)-(33), (37)-(40) into (22) and simplifying the result gives the desired result (10).

\section{References}

[1] V.Tarokh, N.Seshadri, and A.R.Calderbank, "Space-time codes for high data rate wireless communication: performance criterion and code construction," IEEE Transactions on Information Theory, vol.44, no.2, pp.744-765, March, 1998. Article (CrossRef Link)

[2] S.M.Alamouti, "A simple transmit diversity technique for wireless communications," IEEE Journal on Selected Areas in Communications, vol.16, no.8, pp.1451-1458, October, 1998. Article (CrossRef Link)

[3] A.Sendonaris, E.Erkip, and B.Aazhang, "User cooperation diversity-part II: implementation aspects and performance analysis," IEEE Transactions on Communication, vol.51, no.11, pp.1939-1948, November, 2003. Article (CrossRef Link)

[4] J.N.Laneman and G.W.Wornell, "Distributed space-time coded protocols for exploiting cooperative diversity in wireless networks," IEEE Transactions on Information Theory, vol.49, no.10, pp.2415-2525, October, 2003. Article (CrossRef Link)

[5] T.Cover and A.Gamal, "Capacity theorems for the relay channel," IEEE Transactions on Information Theory, vol.25, no.5, pp.572-584, September, 1979. Article (CrossRef Link)

[6] J.N.Laneman and G.W.Wornell, "Exploiting distributed spatial diversity in wireless networks," in Proc. of 2000 Allerton Conference on Communication, Control and computing, pp.1-10, October, 2000. Article (CrossRef Link)

[7] J.N.Laneman, D.N.Tse, and G.W.Wornell, "Cooperative diversity in wireless networks: efficient protocols and outage behavior," IEEE Transactions on Information Theory, vol.50, no.12, pp.3062-3080, December, 2004. Article (CrossRef Link)

[8] Y. Wu, P. A. Chou, and S.-Y. Kung, "Information exchange in wireless networks with network coding and physical-layer broadcast," in Proc. of 2005 Conference on Information Science and Systems, pp.1-7, March 16-18, 2005. Article (CrossRef Link)

[9] P. Popovski and H.Yomo, "Bi-directional amplification of throughout in a wireless multi-hop network," in Proc. of IEEE 63th Vehicular Technology, pp.7-10, May 7-10, 2006. Article (CrossRef Link)

[10] B. Rankov and A. Wittneben, "Spectral efficient protocols for half-duplex fading relay channels," IEEE Journal on Selected Areas in Communications, vol.25, no.2, pp.379-389, February, 2007. Article (CrossRef Link)

[11] R.Ahlswede, N.Cai, S.-Y.R.Li, and R.W.Yeung, "Network information flow," IEEE Transactions on Information Technology, vol.46, no.4, pp.1204-1216, July, 2000. Article (CrossRef Link)

[12] S.Kim, P.Mitran, and V.Tarokh, "Performance bounds for bi-directional relaying," IEEE Transactions on Information Theory, vol.54, no.11, pp.5235-5241, November, 2008. Article (CrossRef Link)

[13] Y.Han, S.H.Ting, C.K.Ho, and W.H.Chin, "Performance bounds for two-way amplify-and-forward relaying," IEEE Transactions on Wireless Communication, vol.8, no.1, pp.432-438, January, 2009. Article (CrossRef Link)

[14] Q.Li, S.Ting, A.Pandharipande, and Y.Han, "Adaptive two-way relaying and outage analysis," IEEE Transactions on Wireless Communication, vol.8, no.6, pp.3288-3299, June, 2009. Article (CrossRef Link)

[15] Haijun Zhang, Xiaoli Chu, Weisi Guo, and Siyi Wang, "Coexistence of Wi-Fi and heterogeneous small cell networks sharing unlicensed spectrum," IEEE Communications Magazine, vol.53, no.3, pp.158-164, March, 2015. Article (CrossRef Link)

[16] Haijun Zhang, Chunxiao Jiang, Xiaoli Chu, and Xianbin Wang, "Resource allocation for cognitive small cell networks: a cooperative bargaining game theoretic approach," IEEE Transactions on Wireless Communications, vol.14, no.6, pp3481-3493, June, 2015. Article (CrossRef Link) 
[17] Mingjie Feng, Tao Jiang, Da Chen and Shiwen Mao, "Cooperative small cell networks: high capacity for hotspots with interference mitigation," IEEE Wireless Communications, vol. 21, no. 6, pp.108-116, December, 2014. Article (CrossRef Link)

[18] Xiangming Li, Tao Jiang and Jianping An, "Cooperative Communications Based on Rateless Network Coding in Distributed MIMO Systems," IEEE Wireless Communications, vol.17, no.3, pp.60-67, June, 2010. Article (CrossRef Link)

[19] C. Zhong, S. Jin, and K. K. Wong, "Dual-hop system with noisy relay and interference-limited destination," IEEE Transactions on Communications, vol.58, no.3, pp.764-768, March, 2010. Article (CrossRef Link)

[20] D. Lee and J. H. Lee, "Outage probability for dual-hop relaying systems with multiple interferers over Rayleigh fading channels," IEEE Transactions on Vehicular Technology, vol.60, no.1, pp.333-338, October, 2011. Article (CrossRef Link)

[21] J. B. Kim and D. Kim, "Exact and closed-form outage probability of opportunistic decode-and-forward relaying with unequal-power interferers," IEEE Transactions on Wireless Communications, vol.9, no.12, pp.3601-3606, October, 2010. Article (CrossRef Link)

[22] D. Lee and J. H. Lee, "Outage probability for opportunistic relaying on multi-cell environments," in Proc. of IEEE 69th Vehicular Technology Conference, pp.1-5, April 26-29, 2009. Article (CrossRef Link)

[23] Youyun $\mathrm{Xu}, \mathrm{Xiaochen} \mathrm{Xia}$ and $\mathrm{Kui} \mathrm{Xu}$, "Symbol error rate of two-way decode and forward relaying with co-channel interference," in Proc. of 2013 IEEE 24th International Symposium on Personal Indoor and Mobile Radio Communications, pp.138-143, September 8-11, 2013. Article (CrossRef Link)

[24] Sajad Hatamnia, Saeed Vahidian, and Mahmoud Ahmadian-Attari, "Performance analysis of two-way decode and forward relaying in the presence of co-channel interferences," IET Communications, vol.8, no.18, pp.3349-3356, December, 2014. Article (CrossRef Link)

[25] Haijun Zhang, Chunxiao Jiang, Xiaotao Mao, and Hsiao-Hwa Chen, "Interference-limited resource optimization in cognitive femtocells with fairness and imperfect spectrum sensing," IEEE Transactions on Vehicular Technology, doi:10.1109/TVT.2015.2405538, 2015. Article (CrossRef Link)

[26] E. Soleimani-Nasab, M. Matthaiou, M. Ardebilipour and G. K. Karragianadis, "Two-way AF relaying in the presecne of co-channel interference," IEEE Transactions on Communications, vol. 61, no. 8, pp.3156-3169, August. 2013. Article (CrossRef Link)

[27] E. Soleimani-Nasab, M. Matthaiou, G. K. Karagiannidis and M. Ardebilipour, "Two-way interference-limited AF relaying over Nakagami-m fading channels," in Proc. of 2013 IEEE Global Communications Conference (GLOBECOM), pp.4275-4281, December 9-13, 2013. Article (CrossRef Link)

[28] E. Soleimani-Nasab, M. Matthaiou and G. K. Karagiannidis, "Two-way interference-limited AF relaying with selection-combining," in Proc. of 2013 IEEE International Conference on Acoustics, Speech and Signal Processing (ICASSP), pp.4992-4996, May 26-31, 2013.

Article (CrossRef Link)

[29] Daniel Benevides da Costa, Haiyang Ding, and Jianhua Ge, "Interference-limited relaying transmissions in dual-hop cooperative networks over Nakagami-m fading," IEEE Communications Letters, pp.503-505, March, 2011. Article (CrossRef Link)

[30] Kais Ben Fredj, Salama S Ikki, and Sonia Aissa, "Performance analysis of two-way opportunistic decode and forward based systems in Nakagami-m fading environments," IET Communications, vol.8, no.9, pp.1626-1636, June, 2014. Article (CrossRef Link)

[31] P.Popovski and H.Yomo, "Physical network coding in two-way wireless relay channels," in Proc. of 2007 IEEE International Conference on Communications, pp.707-712, June 24-28, 2007. Article (CrossRef Link)

[32] W. Nam, S.-Y. Chung, and Y. H. Lee, "Capacity bounds for two-way relay channels," in Proc. of 2008 IEEE International Zurich Seminar on Communications, pp.144-147, March 12-14, 2008. Article (CrossRef Link)

[33] Y.-D. YAO and A. Sheikh, "Outage probability analysis for microcell mobile radio systems with 
cochannel interferers in Ricaian/Rayleigh fading environment," IEE Electronics Letters, vol.26, no.13, pp.864-866, June, 1990. Article (CrossRef Link)

[34] M.Nakagami, "The m-distribution-a general formula of intensity distribution of rapid fading," in proc. of Statistical Methods in Radio Wave Propagation symposium, pp.3-6, June 18-20, 1960. Article (CrossRef Link)

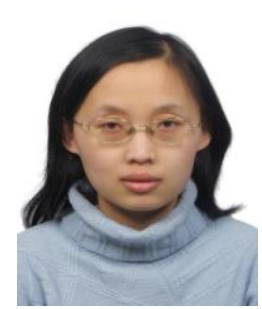

Jinhong Fan received her BS degree from Taiyuan University of Technology, China, in 2001. She is currently a Ph.D. candidate with School of Information and Communication Engineering, Beijing University of Posts and Telecommunications, Beijing, China. Her research interests include cooperative users and relay systems for wireless communication and MIMO systems.

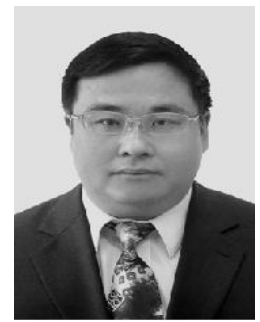

Chaowei Yuan is currently a professor of with School of Information and Communication Engineering, Beijing University of Posts and Telecommunications, Beijing, China. His research interests include wireless communication and mobile communication. 